\title{
LA PERIFERIA IZQUIERDA DE LA PALABRA: PREFIJOS Y ELEMENTOS COMPOSITIVOS
}

\author{
THE LEFT PERIPHERY OF WORDS: \\ PREFIXES AND COMBINING FORMS \\ Josefa Martín García \\ Universidad Autónoma de Madrid \\ josefa.martin@uam.es
}

\begin{abstract}
Resumen: En este trabajo se analizan las diferencias entre los elementos compositivos y los prefijos. Concretamente, defendemos que los elementos compositivos funcionan como nombres, por lo cual pueden ser argumentos y combinarse con sufijos. Los prefijos, en cambio, presentan significados menos precisos como consecuencia de la pérdida de rasgos léxicos, lo que les permite convertirse en operadores y constituir escalas semánticas. Puesto que este proceso de gramaticalización se da en distinto grado, los prefijos no presentan un comportamiento homogéneo.

Palabras clave: prefijación; composición; gramaticalización; morfología léxica; derivación.
\end{abstract}

Abstract: This paper examines in detail the differences between combining forms and prefixes. Specifically, it is argued that combining forms function as nouns and therefore can be arguments and combine with suffixes. On the other hand, prefixes show less specific meanings as a consequence of the loss of lexical features, which allows them to become operators and form semantic scales. Since this grammaticalization process occurs in differing degrees, prefixes do not show an homogeneous behaviour.

Keywords: prefixation; compounding; grammaticalization; lexical morphology; derivation.

Recepción: 20 de septiembre de 2018; aceptación: 29 de mayo de 2019. 
INTRODUCCIÓN*

En la posición izquierda de la palabra, pueden aparecer distintos elementos para dar lugar a una formación morfológica compleja. Tales elementos representan distintas categorías que van desde las raíces, como unidades independientes (ciudad-dormitorio, ético-moral), hasta los prefijos, como unidades dependientes (in-moral, des-hacer). Entre los dos extremos se sitúan elementos dependientes de distinta naturaleza que oscilan entre los que están más próximos a las raíces (hidro-terapia), denominados elementos compositivos ${ }^{1}$, o a los prefijos (macrofiesta). De forma específica, entre las unidades independientes se incluyen raíces con cierta recurrencia (videojuego, videoaficionado, videoconferencia) o sin ella (poeta-pintor, sordomudo) y raíces que adoptan formas ligadas semejantes a los elementos compositivos con vocal final-i/-o (pelirrojo, insecticida, drogodependiente, musicólogo). Las unidades dependientes incluyen elementos compositivos (fotoprotección, termoestable), elementos acortados (ecotasa, eurodiputado) y prefijos (impago, rebonito, predecir).

Desde el punto de vista del proceso de formación, las unidades complejas constituidas por raíces y por elementos compositivos han sido consideradas como compuestos, mientras que las formaciones con prefijos se incluyen dentro de las formas derivadas. No obstante, el problema aparece cuando algunos elementos dependientes comparten propiedades con los elementos compositivos y con los prefijos, o con las raíces y con los prefijos, pues ha provocado muchos desajustes a la hora de establecer la lista y la clasificación tanto de los elementos compositivos como de los prefijos. La naturaleza categorial de estas unidades es relevante independientemente del modelo teórico que se adopte. Los modelos que reconocen la morfología como

Este trabajo se ha elaborado como parte del proyecto de investigación "Microparámetros en las interficies de la sintaxis con otros componentes del sistema lingüístico. Variación gramatical y variedades del español” (FFI2017-87140-C4-4-P), financiado por el Ministerio de Ciencia, Innovación y Universidades del Gobierno de España. Expreso mi agradecimiento a los revisores anónimos de la Nueva Revista de Filología Hispánica por sus valiosos comentarios.

${ }^{1}$ Los elementos compositivos, como los denominaremos en este artículo, han recibido otras denominaciones en los estudios de morfología. Algunas ponen de relieve su origen grecolatino (raíces cultas o grecolatinas, temas neoclásicos o cultos); otras señalan su carácter híbrido entre palabra y prefijo (semipalabra, seudoprefijo, prefijoide). 
un componente autónomo se interesan en la delimitación de los procesos morfológicos, concretamente entre la composición y la derivación. Los modelos en los que existe un único sistema computacional, por el contrario, se centran en fijar la categoría de los elementos situados a la izquierda, que consideran raíces, o bien, núcleos funcionales.

En los trabajos dedicados al tema, se han señalado con frecuencia distintas propiedades de los elementos en cuestión para clasificarlos. Con respecto a los prefijos, Kornfeld (2009) presenta siete tipos a partir de seis pruebas sintácticas con el fin de distinguir entre las unidades que tienen más y menos propiedades de afijos. Montermini (2008) y Rio-Torto (2013 y 2014) hablan de las propiedades prototípicas de los prefijos, es decir, de las cualidades que hacen de un elemento un prefijo. En estas clasificaciones se pone de relieve que los prefijos con menos propiedades de afijos son los que se confundirán con las raíces y con los elementos compositivos. Se han señalado, asimismo, distintos tipos de elementos compositivos. Así, Bok-Bennema (1993), con sus propios términos, reconoce tres clases: prefijoides clásicos combinados con otros elementos cultos (autómata, bibliófilo), prefijoides semi-nativos combinados con palabras (autoservicio, hidromasaje) y prefijoides nativos con una forma independiente semejante (autopista, drogodependiente, euroche$q u e$ ). Iglesias Cancela (2017) también presenta su propia clasificación de los elementos compositivos en la que se diferencian los temas grecolatinos (acanto-, crono-) de los temas acortados (buco-, italo-). Otros, como Gonçalves y Emmerick (2012), reconocen distintas unidades intermedias (afijoide, splinter, radical neoclásico, radical ligado) en un continuo que va desde el afijo hasta el radical libre, a partir del origen (clásico/ no clásico) y de la forma (acortamiento/ raíces cultas).

A diferencia de las propuestas anteriores, defendemos en este trabajo dos únicas categorías para distinguir los elementos dependientes que aparecen en la posición izquierda de la palabra: elementos compositivos y prefijos. Ambas unidades morfológicas son modificadores, pero se diferencian en el modo de modificar la base a la que se adjuntan: los elementos compositivos modifican como sustantivos; los prefijos, en cambio, como adjetivos, adverbios, preposiciones y cuantificadores. De esta diferencia crucial se deriva el distinto comportamiento que presentan los elementos compositivos y los prefijos, caracterizado por diversas propiedades que se han dado para clasificar 
estos elementos. Asimismo, sostenemos que los prefijos no presentan un comportamiento homogéneo, puesto que tienen un significado poco especificado como consecuencia de la pérdida de rasgos léxicos, que se da en distinto grado. Hemos prescindido en nuestro estudio de los elementos compositivos finales, a diferencia de otros estudios, pues los elementos que aparecen en la posición final tienen propiedades distintas de las que caracterizan a los elementos situados en la periferia izquierda de la palabra: fundamentalmente, son categorizadores y tónicos (o bien fijan el acento de la palabra resultante). Consideramos, así, que la posición de un constituyente en la estructura interna de la palabra es determinante para su funcionamiento.

Hemos dividido el trabajo en dos apartados. En el primero, estudiamos la relación que mantienen los elementos dependientes iniciales con el constituyente al que se adjuntan. En el segundo, establecemos la diferencia entre los elementos compositivos y los prefijos a partir de once propiedades, las cuales ponen de relieve el distinto comportamiento de los prefijos y su indeterminación semántica a causa de la pérdida de rasgos léxicos. Cerramos el artículo con algunas conclusiones sobre la prefijación como proceso morfológico.

\section{MODIFICACIÓN A LA IZQUIERdA DE LA PALABRA}

En las palabras complejas, los elementos situados en el margen izquierdo pueden ser núcleos de la formación, como en camposanto o ciudad-dormitorio, o bien modificadores, en cuyo caso el núcleo es el constituyente de la derecha (vasodilatador, videovigilancia).

En su función de modificadores, las unidades iniciales tienden a ser elementos dependientes y a fundirse con el núcleo, como muestran las raíces que pierden la vocal final y adoptan la marca vocálica $-i$ y $-o$, lo que las asemeja a los elementos compositivos ( pelo + rojo > peli-rojo; mano + atar $>$ maniatar $)$. Lo mismo ocurre en las raíces de los compuestos híbridos, en los que el elemento compositivo de la derecha es el núcleo de la formación (insecto + -cida > insecti-cida; cocaína + -mano > cocainó-mano). En estos casos, el primer elemento pierde su acento, dado que el núcleo del compuesto mantiene la sílaba tónica (pelirrojo, insecticida), o bien, en el caso de los compuestos híbridos, el elemento compositivo nuclear fija el acento de la unidad compleja 
(cocainómano). La posición izquierda de la palabra favorece también el acortamiento de las unidades, que pasan a ser elementos dependientes y se fusionan con el de la derecha, como ocurre en los compuestos coordinados (social + laboral $>$ sociolaboral; biológico + social > biosocial) o en los compuestos formados por gentilicios (hispanofrancés/ francoespañol; hispanoitaliano/ italoespañol). Estas mismas características se dan, asimismo, en los acortamientos de adjetivos relacionales: tasa ecológica $>$ ecotasa; diputado europeo > eurodiputado. Como vemos, los elementos situados a la izquierda de la palabra tienden a identificarse formalmente mediante formas más simples y mediante las vocales finales $-i /-o$, lo que incrementa la recurrencia en las formaciones y, en consecuencia, su productividad.

A diferencia de las raíces, que pueden ser también núcleos, los elementos dependientes iniciales sólo funcionan en la palabra compleja como modificadores y, por tanto, nunca imponen su categoría gramatical. Pueden modificar al núcleo como sustantivos (hidroterapia = 'terapia por medio del agua'), como adjetivos ( macroconcierto $=$ 'concierto grande'), como adverbios ( semienterrado = 'enterrado parcialmente'), como preposiciones ( sobrevolar $=$ 'volar sobre') o como cuantificadores (bidimensional = 'de dos dimensiones'). A partir de la modificación de la base, es posible distinguir dos categorías de elementos dependientes iniciales: los elementos compositivos y los prefijos. La modificación como sustantivo es exclusiva de los elementos compositivos; los prefijos, en cambio, pueden modificar como adjetivos, adverbios, preposiciones y cuantificadores.

En la modificación como sustantivos, los elementos compositivos se unen al núcleo para establecer distintas relaciones sintáctico-semánticas, según la naturaleza gramatical del núcleo y del contenido semántico del propio elemento dependiente, el cual puede indicar la causa $(1 a)$, el paciente $(1 b)$, el instrumento $(1 c)$ o el medio $(1 d)$ :

(1) a. termoelectricidad = 'electricidad producida por el calor';

b. dermoprotector = 'que protege la piel';

c. hidromasaje = 'masaje realizado con agua';

d. aerotransportar = 'transportar por el aire'.

Como modificadores adjetivos, los prefijos se unen a núcleos nominales y funcionan como adjetivos calificativos (seudonoticia $=$ 'noticia falsa') o relacionales (eurodiputado $=$ 'diputado euro- 
peo'). En el primer caso, los prefijos pueden indicar distintas propiedades del sustantivo base:

(2) a. macrofiesta ('fiesta grande'), microespacio ('espacio de dimensiones reducidas');

b. superordenador ('ordenador bueno'), infravivienda ('vivienda de malas condiciones');

c. neocapitalismo ('capitalismo nuevo, contemporáneo'), paleoclima ('clima de épocas geológicas antiguas');

d. seudoproblema ('problema falso').

Los elementos acortados procedentes de adjetivos relacionales han desarrollado funciones prefijales en su uso como clasificadores del sustantivo al que modifican (eurocheque, ecoturismo, bioagricultura). Cuando la forma acortada coincide con el elemento compositivo, las dos unidades se diferencian por el significado. Por ejemplo, el prefijo eco-de una formación como ecoetiqueta exhibe un contenido significativo distinto del que corresponde al elemento eco- en economía: en el primer caso, se refiere al adjetivo ecológico y, en el segundo, al significado originario 'casa'. Lo mismo ocurre con bio- en formaciones como biosensor o bioagricultura, en las que bio-no puede parafrasearse como 'vida', sino como 'biológico': 'sensor de material biológico', 'agricultura biológica', respectivamente.

Los prefijos también pueden modificar la base a la que se adjuntan como adverbios. En este caso, las bases seleccionadas son verbos $(3 a)$, adjetivos $(3 b)$ y adverbios $(3 c)$, y las formaciones resultantes indican fundamentalmente contenidos intensivos y negativos:

(3) a. sobrecargar, remirar, ultracongelar, desconocer, prejubilar;

$b$. archiconocido, superbarato, infravalorado, inmaduro;

c. supercerca, relejos.

Otra posibilidad es que los prefijos funcionen como preposiciones. De hecho, algunos presentan la misma forma que las preposiciones (4), y otros, el mismo significado de alguna preposición o locución preposicional, pero con una forma distinta (5):

(4) a. entretela = 'tela colocada entre dos telas';

b. sobrevolar = 'volar sobre'; 
(5) a. [crema] antiarrugas = 'contra las arrugas';

b. [manifiesto] pro escuela pública = 'a favor de la escuela pública';

c. subtítulo = 'título debajo de otro título'.

Sin embargo, algunos prefijos preposicionales pueden modificar adjetivos y verbos con valores de intensidad, como hacen los prefijos adverbiales: entrecano = 'medio cano', sobrecargar $=$ 'cargar mucho'.

Por último, los prefijos pueden modificar la base como cuantificadores de distinto tipo: precisos $(6 a)$, imprecisos $(6 b)$, fraccionarios $(6 c)$ :

(6) $a .\{$ mono/ bi/ tri...\} color;

b. policultivo, multiprocesador, pluriempleo;

c. miligramo, centímetro, nanogramo.

En este apartado, hemos establecido una primera diferencia entre los elementos compositivos y los prefijos según su modo de modificar en cuanto elementos dependientes e iniciales. Esta diferencia es decisiva para entender el funcionamiento de los elementos compositivos y de los prefijos, como tratamos de mostrar en el apartado siguiente.

\section{Prefijos y Elementos Compositivos}

\section{Propiedades de los elementos dependientes iniciales}

Como sugiere el título de este apartado, los elementos compositivos y los prefijos tienen en común dos propiedades: están situados al inicio de la palabra y son unidades dependientes. Más allá de estas dos cualidades, cada unidad exhibe características específicas que la identifican como prefijo o como elemento compositivo. En distintos trabajos sobre prefijación (entre otros, Iacobini 1997; Prćić 2005; Montermini 2008; RAE y ASALE 2009; Kornfeld 2009; Rio-Torto 2014; Martín García 2017), se han propuesto características para definir los prefijos. Partiendo de estas propiedades, diferenciaremos los prefijos de los elementos compositivos y, al mismo tiempo, mostraremos que estas diferencias se derivan del modo en que cada una de las dos categorías modifica la base a la que se adjunta. 
Modificación y endocentricidad. Tanto los prefijos como los elementos compositivos se subordinan al constituyente situado a la derecha, por lo cual no se erigen como núcleo de la formación ni son elementos categorizadores, a diferencia de lo que ocurre cuando un elemento compositivo aparece al final de la palabra: así, el formante logo es núcleo en filólogo y modificador en logopedia. Las construcciones con elementos compositivos a la izquierda o con prefijos son endocéntricas con núcleo a la derecha, por lo que se distinguen de los compuestos formados por raíces cuyo núcleo está a la izquierda (ciudad-dormitorio, coche eléctrico), el mismo orden que se da en las estructuras sintácticas. Asimismo, las formaciones con elementos compositivos o con prefijos contravienen la posición nuclear de las estructuras sintagmáticas construidas con los mismos formantes, pues en éstas el núcleo aparece a la izquierda (tasa ecológica/ ecotasa; protector de la piel/ dermoprotector).

En suma, los elementos compositivos y los prefijos son modificadores. Como hemos señalado en el apartado anterior, las dos categorías se diferencian en el modo de modificar la base: los elementos compositivos modifican como sustantivos; los prefijos, por el contrario, como adjetivos, adverbios, preposiciones o cuantificadores.

Elementos átonos. Los elementos compositivos y los prefijos están constituidos por una o dos sílabas (generalmente, no trabadas) y son átonos: propiedades que facilitan su fusión con la base. En las formaciones resultantes se conserva el acento del núcleo (sub-tropicál, seudo-ciéncia, eco-tása, hidro-terápia). No obstante, estas mismas unidades pueden ser tónicas cuando se constituyen como formas independientes: gasolina súper, sueldo extra, vida eco.

Recursividad. A diferencia de los constituyentes dependientes finales, los elementos compositivos iniciales y los prefijos pueden reduplicarse $(7 a)$, combinarse con otros elementos de su misma categoría $(7 b)$ e incluso coordinarse $(7 c)$ :

(7) a. súper-superfamoso, anti-anti-capitalismo;

b. súper-mega-famoso, hidrobiológico;

c. macro y microeconomía, mono y bicolor.

La combinación de un mismo elemento se da sólo con los prefijos que indican intensidad, de modo que la reduplicación 
supone un grado mayor del contenido del núcleo (súper-superfamoso; macro-macroconcierto), o con los prefijos que expresan oposición para presentar con la reduplicación un contenido contrario (anti-anti-capitalismo; contra-contra-aviso). No obstante, en las páginas de Internet es posible encontrar formaciones neológicas en las que se reduplican también las formas procedentes de adjetivos relacionales acortados. Por ejemplo, la reduplicación de eco- o ciber- se utiliza para denominar con más énfasis determinados establecimientos (Eco-ecotienda; Ecoeco centro de bienestar, ciber-cibercafé) o distintas realidades de la Red (Eco-ecomagazines; ciber-ciber perfil). La posibilidad de repetir una misma unidad está más limitada con los elementos compositivos.

En cuanto a la combinación de unidades, los prefijos intensivos presentan cierta recursividad (súper-mega-famoso, ultrasúper-conocido) con algunas restricciones (Martín García 1998). Encontramos también secuencias altamente productivas con prefijos preposicionales y adverbiales (des-em-barcar, re-em-barcar). En el caso de los elementos compositivos, son posibles algunas combinaciones, sobre todo en las lenguas de especialidad (hidro-bio-lógico, geo-crono-logía).

Más frecuente que la combinación de los prefijos y de los elementos compositivos por yuxtaposición es la coordinación de distintas formas. Se coordinan los prefijos que funcionan como adjetivos calificativos con significado contrario ( macro y microeconomía, neo y paleomarxistas), los prefijos cuantificadores (mono y bicolor, bi y multilateral) o los prefijos provenientes del acortamiento de adjetivos relacionales (eco y bioservicios, cibery tecnocultura). También hay coordinación de algunos elementos compositivos (hidro y aerodinámico, hidro y termoelectricidad).

Combinación con raices y elementos compositivos. Como hemos visto ya en ejemplos anteriores, los elementos compositivos y los prefijos, combinados con raíces, forman nuevas palabras. Otra posibilidad es que los prefijos ( $8 a$ y $b$ ) y los elementos compositivos $(8 c)$ se adjunten a elementos compositivos finales para dar lugar a nuevas formaciones:

(8) a. uníparo, bicéfalo, multiforme;

$b$. neólogo, microcéfalo, homofonía;

c. hidrófilo, termófono, geofagia. 
En estos ejemplos se incluyen prefijos que modifican como cuantificadores $(8 a)$ o como adjetivos $(8 b)$. Los elementos compositivos iniciales de $(8 c)$ funcionan como sustantivos subordinados al elemento final.

Múltiple selección categorial. Los elementos compositivos se adjuntan a sustantivos (hidroelectricidad, termoterapia) y a adjetivos (fotodegradable, neurotransmisor) manteniendo su modificación como sustantivos. A diferencia de ellos, los prefijos tienden a seleccionar bases de distintas categorías: entretela, entremezclar, entrecano; deshonra, desleal, deshacer, superhombre, superabundar, superbarato, supercerca. Como consecuencia, un mismo prefijo puede alterar su modo de modificar. Por ejemplo, los que seleccionan bases sustantivas actúan como modificadores adjetivos de forma muy productiva (seudociencia, microtarjeta, megaciudad, neocatolicismo). No obstante, algunos prefijos adjetivales han ampliado sus posibilidades de selección y pueden adjuntarse a verbos (9) y a adjetivos (10):

(9) a. Aunque os tenga que llevar a todos, yo quiero macroquedar (https://www.spaniards.es/foros/contactos/que-pasanennnnnnns-de-miami, consultado el 5 de septiembre de 2018);

b. Espero pseudo-comprar un techo dentro de 2 años (https://m.forocoches.com/foro/showthread. php? $\mathrm{t}=1589206 \&$ page $=4$, consultado el 5 de septiembre de 2018);

c. Microinyectar (DEA).

(10) a. relaciones pseudoconcretas $(D E A)$;

b. aspectos macroestéticos $(D E A)$;

c. tejidos neoformados $(D E A)$.

Este mismo comportamiento se observa con los prefijos procedentes de adjetivos relacionales acortados, los cuales son muy productivos unidos a sustantivos (ecotransporte, tecnoviaje, ciberterrorismo), pero también pueden seleccionar verbos (11) y adjetivos (12):

(11) a. Aprenden a ecodiseñar envases para una economía circular (https://www.laverdad.es/ababol/ciencia/aprenden-ecodisenar-envases-20180320003829-ntvo.html, consultado el 5 de septiembre de 2018); 
b. Estoy listo para tecnoestudiar (http://tecnobabil.blogspot.com/2013/02/la-madera-2-eso.html, consultado el 5 de septiembre de 2018);

c. Gracias por este foro que me ha permitido ciberconectar con lo mío (http:/ / miradas3-personajessomoza.blogspot. com/, consultado el 5 de septiembre de 2018).

(12) a. fábrica ecosostenible (http://www.widex.es/fabrica-ecosostenible, 5 de septiembre de 2018);

$b$. verano tecnodivertido (https://norte-monterrey. vlex.com.mx/vid/x00a1-tener-verano-tecnodivertido-78237191, consultado el 5 de septiembre de 2018);

c. He conocido gente ciberinteresante (https://www.zonanegativa.com/zona-negativa-entrevista-a-kenny-ruiz/, consultado el 5 de septiembre de 2018).

Este paralelismo -y otros que se irán señalando- entre el funcionamiento de los prefijos que modifican como adjetivos calificativos y las formas acortadas procedentes de adjetivos relacionales justifica que estas últimas sean consideradas prefijos.

Polisemia. Dado que los elementos compositivos y los prefijos no funcionan como núcleo de la formación y, por tanto, no son el constituyente significativo principal, cabría esperar que estas formas establecieran con la base a la que se subordinan distintas relaciones semánticas. Sin embargo, esta observación sólo es válida para los prefijos, pues los elementos compositivos presentan un significado constante y específico. Los prefijos muestran contenidos significativos más imprecisos y generales, hecho que provoca que estos elementos sean polisémicos a causa del desarrollo de nuevos matices semánticos en su combinación con la base. Por ejemplo, algunos prefijos pueden tener significados locativos e intensivos (sobrevolar/ sobrecargar, entremezclar/ entreabrir, subtítulo/ subdesarrollado), iterativos e intensivos (rehacer/ rebuscar), o bien de cualidad y de cuantificación (megaciudad/ megavatio; nanotecnología/ nanosegundo). Otros prefijos oscilan entre el significado locativo y el temporal (precordillera/ preaviso; postdorso/ posguerra); incluso el significado general de negación (desobedecer) puede admitir distintos valores, como la reversión (deshacer) o la privación (desnatar). Este comportamiento se da en prefijos de distinto tipo.

El prefijo adjetival macro- tiene el significado general de 'grande', que adquiere diversos matices significativos según el 
sustantivo al que modifique. Por ejemplo, en formaciones como macroconcierto o macroencuesta, macro- alude a las dimensiones físicas de la entidad, pero también puede hacer referencia a la dimensión de las entidades con las que el sustantivo base está relacionado: macrofotografía no es una 'fotografía grande', sino una 'fotografía con imagen grande o ampliada'; macrofauna no es un 'conjunto grande de animales', sino la 'fauna de animales grandes'. De este significado general, macro- puede derivar al significado de 'global, general', presente en macroestructura o macroeconomía, o incluso puede denotar la pluralidad de entidades como los prefijos cuantificadores: macroinstrucción es una "secuencia de instrucciones que se realizan automáticamente con una sola orden" (DLE).

Los prefijos procedentes de adjetivos relacionales acortados han desarrollado también nuevos significados que, en algunos casos, permiten diferenciar la construcción morfológica de la formación sintagmática. Así, eco-, acortamiento de ecológico, tiene el significado general de 'relacionado con el medio ambiente y con la naturaleza', a partir del cual surgen distintos matices: 'no perjudicial para el medio ambiente' (ecotransporte, ecocombustible); 'que defiende, protege el medioambiente' (ecotasa, ecoeducación); 'en la naturaleza' (ecoturismo, ecociclismo); 'sin productos químicos' (ecofertilizante, ecopastelería). Cuando eco- se une a adjetivos o a verbos, aporta significados adverbiales relacionados con los anteriores (ecoconstruir, ecosostenible), que están excluidos de la construcción sintáctica: ecocaminar es 'caminar en el medio natural', pero es poco probable que se acepte la construcción sintáctica correspondiente ??caminar ecológicamente.

Los prefijos cuantificadores se caracterizan por indicar el número de entidades que denota la base (monocarril, bicolor, multicanal). Sin embargo, a partir de este significado general pueden derivarse otros valores. Tal es el caso de mono- ('uno'), que puede expresar el significado de 'simple, no divisible' en lenguajes de especialidad (monocristal, monosacárido). Otros prefijos cuantificadores han derivado hacia significados intensivos, como semi-, cuantificador en formaciones como semicírculo o semieje, que también presenta el contenido intensivo de "casi, parcialmente' en semienterrado o semidestruir.

Productividad. El hecho de que los prefijos tengan un significado más general y una selección categorial más amplia hace 
que estos elementos sean más productivos, como los prefijos cuantificadores (13a), preposicionales (13b), adverbiales $(13 c)$, adjetivales calificativos $(13 d)$ y adjetivales relacionales $(13 e)$. Los elementos compositivos tienen una productividad más reducida $(13 f)^{2}$ :

(13) a. multicanal, multicelular, multicéntrico, multicolor, multicultural, multidimensional;

b. sobrearco, sobreático, sobreceja, sobreedificar, sobrefalda, sobreimprimir;

c. relimpio, rebarato, rebonito, rebuscar, remirar, requemar;

d. minibar, minibasket, minibús, minicadena, minicumbre, minifalda;

e. eurocámara, eurocentrismo, eurocheque, eurocomisario, eurocomunismo, euroconector;

f. hidroavión, hidrobiología, hidroelectricidad, hidrofobia, hidrófugo, hidrogel.

Escalas semánticas. Algunos prefijos con significado más general establecen relaciones con otros para constituir escalas semánticas de distinto tipo: relaciones de oposición (pre-/ pos-, mini-/ maxi-; anti-/ pro-); relaciones de reversión (en-/ des-); relaciones de gradación (macro-, maxi-, mega-/ mini-, micro-; ultra-, archi-, súper-); relaciones de serie (mono-, bi-, tri-... multi-). Presentan esta propiedad los prefijos cuantificadores (bi-, multi-), los preposicionales (sobre-, sub-), los adverbiales (mal-, bien-) y los adjetivales que funcionan como calificativos (macro-, micro-, neo-, paleo-). Sin embargo, no tienen tal propiedad los prefijos procedentes de adjetivos relacionales acortados (euro-, eco-, ciber-) ni los elementos compositivos (hidro-, termo-, neuro-).

Operadores. A diferencia de los elementos compositivos, los prefijos presentan varios tipos de modificación -como hemos señalado ya- con que logran incidir en la base de diversas formas, es decir, tienen distinto alcance, como ocurre con los operadores. Por ejemplo, los prefijos pueden afectar a las propiedades de

${ }^{2}$ Adoptamos una idea básica de la productividad de un proceso morfológico basada en el número de formaciones con un mismo elemento. Nos hemos basado solamente en el número de formaciones que incluyen los diccionarios generales de español y en los procesos más productivos. Por ejemplo, en el grupo de los elementos compositivos, hidro- es más productivo que agro-, helio-, termo-, entre muchos otros. 
los complementos del sustantivo y no sólo a las propiedades del sustantivo propiamente dicho. Así, micro- puede hacer referencia a las dimensiones de la entidad representada en la base ( microtarjeta $=$ 'tarjeta pequeña') o de otra entidad con que la base está relacionada (microfotografía = 'fotografía de cosas muy pequeñas'). Del mismo modo, el significado de paleo- es 'antiguo' como en paleoclima, pero se asocia también a los fósiles como objetos muy antiguos estudiados por las ciencias (paleobiología, paleobotánica).

En el caso de los verbos, un elemento como macro- no afecta a la dimensión de los objetos, sino a la cantidad: macrovender no significa 'vender cosas grandes', sino 'vender muchas cosas'. Los prefijos negativos pueden anular completamente la base, como en desconocer, cuyo significado es 'no conocer'; pero en otros casos, la negación sólo del argumento interno se hace posible: así, desinformar no significa 'no informar', sino 'informar con noticias falsas (no noticias)'. Los prefijos preposicionales con bases verbales pueden presentar valores propios de las preposiciones (sobrevolar, contraponer), pero también, en ocasiones, actuar como operadores iterativos que llevan la acción a repetirse. Por ejemplo, sobreimprimir se interpreta como 'imprimir sobre algo ya impreso', es decir, se entiende que hay un resultado de una acción previa sobre el que incide la acción de sobreimprimir.

Los prefijos cuantificadores, por su parte, consiguen establecer el número de entidades representadas en la base (bicolor) o el número de veces en que tiene lugar la base (bicampeón = 'campeón en dos ocasiones'). En otros casos, la cuantificación puede indicar el número de entidades de un sustantivo con el que la base está relacionada: generalmente, su argumento. Así, en una formación como multidonante, multi- no denota el número de entidades de la base ('muchos donantes'), sino el de órganos de un mismo donante.

Argumentos. Los elementos compositivos funcionan como sustantivos, por lo cual pueden establecer distintas relaciones sintáctico-semánticas con el núcleo: causa (termoelectricidad, fotoenvejecimiento, aerogenerador), paciente (dermoprotector, termodifusión), instrumento (hidromasaje, aeroterapia, criocoagulación) o medio (aerotransportar). En algunas formaciones, los elementos compositivos pueden saturar posiciones argumentales del núcleo (14) o establecer otras relaciones semánticas (15): 
(14) a. fotoalergia = 'alergia a la luz';

b. fotoprotector = 'que protege de las radiaciones solares';

c. fotosensible = 'sensible a la luz';

d. hidrosoluble = 'soluble en agua'.

(15) $a$. hidrogel = 'gel constituido por agua';

b. geomagnetismo = 'magnetismo de la tierra (terrestre)'.

Estas mismas relaciones sintáctico-semánticas se dan en las formaciones constituidas por una raíz sustantiva inicial que no funciona como núcleo: pelirrojo, maniatar, drogodependiente, insecticida, musicólogo. Los prefijos, por el contrario, no establecen estos tipos de relaciones.

Combinación con sufijos. Señala Bok-Bennema (1993) que los elementos situados a la izquierda de la palabra son prefijos si son formas dependientes y no pueden combinarse con sufijos. En su clasificación de los prefijoides, esta idea sólo se aplica a los prefijoides nativos -aquellos que se producen por acortamiento y tienen una forma independiente semejante (autopista, drogodependiente). No obstante, la observación de esta autora nos sirve para diferenciar los elementos compositivos de los prefijos. Así, mientras que los elementos compositivos pueden combinarse con sufijos (hídrico, agrario, térmico), los prefijos no admiten esta posibilidad independientemente de la modificación que establezcan con la base: prefijos adjetivales calificativos (*macr-idad, *seud-ico), prefijos adjetivales relacionales $(* e c-o s o$, ${ }^{*}$ ciber-eza), prefijos adverbiales (*super-ificar, ${ }^{*}$ sem-oso) ${ }^{3}$, prefijos preposicionales (*entr-idad, *sobr-izar) o prefijos cuantificadores (*bi-oso, *mult-ico).

En la Tabla 1 presentamos de forma gráfica las propiedades de los elementos dependientes iniciales que acabamos de comentar y las relacionamos con la categoría de estos elementos (compositivos y prefijos) y con la función que desempeñan en la palabra compleja en cuanto elementos modificadores.

${ }^{3}$ Véase que formaciones como malear o maloso no se atienen a este proceso, puesto que no están construidas sobre el adverbio mal, sino sobre el adjetivo malo, como indica su significado: 'hacer que algo sea malo' y 'muy malo', respectivamente. 
TABLA 1

Propiedades de los elementos dependientes iniciales

\begin{tabular}{|c|c|c|c|c|c|c|}
\hline Categoría & $\begin{array}{l}\text { elemento } \\
\text { composi- } \\
\text { tivo }\end{array}$ & prefijo & prefijo & prefijo & prefijo & prefijo \\
\hline Función & nominal & $\begin{array}{l}\text { adjetival } \\
\text { relacional }\end{array}$ & $\begin{array}{l}\text { adjetival } \\
\text { califica- } \\
\text { tivo }\end{array}$ & adverbial & $\begin{array}{l}\text { preposi- } \\
\text { cional }\end{array}$ & $\begin{array}{l}\text { cuanti- } \\
\text { ficador }\end{array}$ \\
\hline $\begin{array}{l}\text { 1) } \\
\text { Modificación y } \\
\text { endocentricidad }\end{array}$ & X & X & X & $\mathrm{X}$ & X & X \\
\hline $\begin{array}{l}\text { 2) } \\
\text { Elementos átonos }\end{array}$ & $\mathrm{X}$ & $\mathrm{X}$ & $\mathrm{X}$ & $\mathrm{X}$ & $\mathrm{X}$ & $\mathrm{X}$ \\
\hline $\begin{array}{l}\text { 3) } \\
\text { Recursividad }\end{array}$ & $\mathrm{X}$ & $X$ & $\mathrm{X}$ & $\mathrm{X}$ & $\mathrm{X}^{4}$ & $\mathrm{X}$ \\
\hline $\begin{array}{l}\text { 4) } \\
\text { Combinación con } \\
\text { raíces y elementos } \\
\text { compositivos }\end{array}$ & $\mathrm{X}$ & $\mathrm{X}$ & $\mathrm{X}$ & $X$ & $\mathrm{X}$ & $\mathrm{X}$ \\
\hline $\begin{array}{l}\text { 5) } \\
\text { Múltiple selección } \\
\text { categorial }\end{array}$ & $\begin{array}{c}\text { sólo N } \\
\text { y A }\end{array}$ & $X$ & $\mathrm{X}$ & $\mathrm{X}$ & $X$ & $\begin{array}{c}\text { sólo N } \\
\text { y A }\end{array}$ \\
\hline $\begin{array}{l}\text { 6) } \\
\text { Polisemia }\end{array}$ & & $\mathrm{X}$ & $\mathrm{X}$ & $X$ & $\mathrm{X}$ & $X$ \\
\hline $\begin{array}{l}\text { 7) } \\
\text { Productividad }\end{array}$ & & $\mathrm{X}$ & $\mathrm{X}$ & $\mathrm{X}$ & $\mathrm{X}$ & $\mathrm{X}$ \\
\hline $\begin{array}{l}\text { 8) } \\
\text { Escalas semánticas }\end{array}$ & & & $\mathrm{X}$ & $\mathrm{X}$ & $\mathrm{X}$ & $\mathrm{X}$ \\
\hline $\begin{array}{l}\text { 9) } \\
\text { Operadores }\end{array}$ & & & $\mathrm{X}$ & $\mathrm{X}$ & $\mathrm{X}$ & $\mathrm{X}$ \\
\hline $\begin{array}{l}\text { 10) } \\
\text { Argumentos }\end{array}$ & $\mathrm{X}$ & & & & & \\
\hline $\begin{array}{l}\text { 11) } \\
\text { Combinación con } \\
\text { sufijos }\end{array}$ & $\mathrm{X}$ & & & & & \\
\hline
\end{tabular}

${ }^{4}$ Con limitaciones.

Nueva Revista de Filología Hispánica (NRFH), LXVIII, 2020, núm. 2, 523-549

ISSN 0185-0121; e-ISSN 2448-6558; DOI: 10.24201/nrfh.v68i2.3649 


\section{La escala funcional}

Como hemos visto hasta el momento, los elementos que pueden aparecer en la posición izquierda de la palabra no se comportan de la misma forma según el modo de modificar la base a la que se adjuntan. Partiendo de las diferencias entre los prefijos y los elementos compositivos, en este apartado consideraremos algunos prefijos con naturaleza menos definida.

Volviendo a la Tabla 1, comentaremos el orden marcado por la función: nominal, adjetival relacional, adjetival calificativo, adverbial, preposicional, cuantificador. Aparecen primero las funciones de modificación que se asemejan más a las categorías léxicas (sustantivo, adjetivo, adverbio), seguidas de las funciones más relacionadas con las categorías gramaticales (preposición, cuantificador). Con esta disposición, se pueden establecer generalizaciones interesantes sobre el comportamiento de las unidades morfológicas que estudiamos.

En primer lugar, los elementos compositivos y los prefijos comparten cuatro propiedades básicas: son modificadores de la base a la que se adjuntan, por lo que nunca serán núcleo de la formación (propiedad 1); son elementos átonos (propiedad 2 ); son elementos recursivos, aunque con ciertas limitaciones en el caso de algunos prefijos (propiedad 3); se combinan con raíces y con elementos compositivos finales (propiedad 4).

En segundo lugar, los elementos compositivos presentan dos propiedades exclusivas que los diferencian de los prefijos: pueden interpretarse como argumentos de la base (propiedad 10) y se combinan con sufijos para dar lugar a formaciones nuevas (propiedad 11).

En tercer lugar, las propiedades de 5 a 9 no se dan en los elementos compositivos y se aplican con cierta vacilación en los distintos tipos de prefijos. Salvo los elementos compositivos y los prefijos cuantificadores, con una selección categorial más limitada, el resto de los prefijos puede seleccionar bases de distintas categorías gramaticales (propiedad 5). Frente a los elementos compositivos, con un significado más específico y con una productividad más restringida, los prefijos exhiben distintos valores significativos (propiedad 6) y son muy productivos (propiedad 7). Dentro del grupo de los prefijos, los que proceden de adjetivos relacionales acortados no establecen escalas semánticas (propiedad 8) ni funcionan como operadores (propiedad 9), a diferencia del resto de los prefijos. 
Según hemos mencionado, los elementos compositivos se diferencian claramente de los prefijos por modificar la base a la que se adjuntan como sustantivos, por tener propiedades exclusivas (propiedades 10-11) y por carecer de otras que sí comparten los prefijos (propiedades 5-9). Esta caracterización acerca los elementos compositivos a las raíces, las cuales presentan un significado léxico más específico y constante, de modo que el grado de polisemia es menor que el que manifiestan los afijos, con un significado más general e impreciso (Olsen 2014). Asimismo, las raíces se combinan de forma natural con sufijos para dar lugar a nuevas palabras (cremoso, montañismo) y pueden constituirse en argumentos (aromaterapia, maniatar). Por todo ello, parece conveniente considerar como compuestos las formaciones con elementos compositivos, semejantes a las formaciones constituidas por raíces. Las palabras prefijadas, en cambio, presentan propiedades diferentes y no son compuestos.

Ahora bien, frente a los elementos compositivos, los prefijos no forman un grupo homogéneo. Así, los prefijos que proceden de adjetivos relacionales (eco-, ciber-, euro-) no establecen escalas semánticas (propiedad 8) ni funcionan como operadores (propiedad 9). En cambio, como el resto de prefijos, presentan cierta polisemia (propiedad 6) y son muy productivos (propiedad 7). De este modo, de las cinco propiedades que definen a los prefijos (propiedades 5-9), son las propiedades 8 y 9 las que se constituyen como las características más definitorias de los prefijos.

El orden adoptado en la Tabla 1, según la modificación de la base, permite diferenciar los elementos compositivos y los prefijos, pero también caracterizar el comportamiento de los prefijos a partir de su función como modificadores. Hemos mencionado que los elementos compositivos modifican como sustantivos, por lo cual pueden ser argumentos. Un prefijo, por el contrario, nunca puede funcionar como un sustantivo ni constituirse como argumento. En el otro extremo, se sitúan los prefijos que modifican como preposición y como cuantificador, dos categorías que carecen de significado léxico. Entre la modificación nominal y la modificación como categorías más gramaticales, se sitúan los prefijos que funcionan como adjetivos y adverbios, dos formas de modificación que pueden darse en un mismo prefijo. En efecto, como hemos visto en el apartado anterior, los prefijos adjetivales han desarrollado usos adverbiales con bases verbales $(16 a)$ y adjetivales $(16 b)$ : 
(16) a. macrovender, seudocomprar, ecoexistir, ciberquedar;

$b$. macroestético, seudoconcreto, ecoagradable, ciberinteresante.

Por su parte, los prefijos preposicionales pueden tener usos adverbiales unidos a verbos (17a) y a adjetivos (17b):

(17) a. sobrecargar, entreabrir, ultracongelar;

b. sobreagudo, entrefino, ultraligero.

Aunque los prefijos son muy productivos en su función como modificadores adverbiales, algunos autores no consideran como prefijos algunos elementos dependientes iniciales. Por ejemplo, Alemany Bolufer (1920), Rainer (1993), Val Álvaro (1999) o Casado Velarde (2015) sostienen que formaciones como malvivir o malherido son compuestos, puesto que mal es adverbio. No obstante, cabe señalar que sólo suelen considerarse bien y mal en los esquemas compositivos adverbio + verbo o adverbio + adjetivo; por tanto, si dichos esquemas son compuestos, no son productivos, hecho que contrasta con la alta productividad de los prefijos como modificadores adverbiales, como en los ejemplos de (18):

(18) $a$. desconfiar = 'no confiar', rebuscar = 'buscar minuciosamente';

b. inmaduro = 'no maduro', rebarato $=$ 'muy barato'.

Respecto a los prefijos adjetivales, es de destacar que la modificación inicial por medio de un adjetivo representa un esquema restringido en la composición, frente al patrón inverso en el que el adjetivo aparece pospuesto al sustantivo. Como señalan Bustos Gisbert (1986) y Val Álvaro (1999), los compuestos adjetivo + nombre quedan reducidos en español a unas pocas series recursivas con medio (media naranja, medialuna) o malo (malaleche, malhumor), las cuales suelen exhibir cierta opacidad semántica, frente al esquema inverso que proporciona mayor número de formaciones (camposanto, aguardiente, coche eléctrico). Por ejemplo, media naranja alude a una persona, no a una parte del fruto; malaleche no significa un tipo de leche, sino más bien una intención. Estos compuestos son distintos de formaciones como seudociencia o megaciudad, que presentan un significado composicional y están formadas con prefijos adjeti- 
vales muy productivos. De hecho, la modificación adjetival de un sustantivo mediante prefijos es muy productiva en español, como se observa en otros prefijos adjetivales: preacuerdo = 'acuerdo anterior', resobrino = 'sobrino segundo'.

Lo dicho hasta el momento justifica que los elementos dependientes iniciales que modifican como adverbios y adjetivos deban considerarse prefijos y no elementos compositivos. Admitir que las palabas formadas por elementos adverbiales y adjetivales son compuestos implicaría asumir procesos de modificación muy poco productivos en la composición y carentes de significado composicional en muchos casos. Asimismo, obligaría a reconocer dos tipos de unidades: uno como elementos compositivos y otro como prefijos, pues los esquemas adjetivo + nombre y adverbio + verbo/ adjetivo existen en la prefijación y son productivos. Por ello, si asumimos que estos elementos son prefijos, podemos explicar estos procesos de formación de manera más sencilla y, a la vez, entender mejor el funcionamiento de los prefijos.

\section{Pérdida de rasgos y gramaticalización}

En los apartados anteriores, hemos señalado que los prefijos contienen un significado menos preciso que los elementos compositivos. Esta característica se ha explicado a partir de la pérdida de rasgos léxicos como proceso de gramaticalización. Concretamente, se han propuesto escalas que van desde el elemento más libre y menos gramatical (el adverbio) al elemento más ligado y más gramatical (el prefijo), pasando por estados intermedios como la preposición o la partícula ${ }^{5}$. Estas escalas permiten reconocer las raíces que pierden sus rasgos más léxicos para funcionar como prefijos ${ }^{6}$. En español, hay varios ejemplos de este proceso.

${ }^{5}$ Por ejemplo, en la escala de gramaticalización que propone Heyna (2012), se añade un estado intermedio entre la preposición y el prefijo: el prefijo separable. Amiot y De Mulder (2002), por su parte, distinguen dos estados intermedios entre el adverbio y el prefijo: la preposición circunstancial y la preposición regida.

${ }^{6}$ Bok-Bennema (1993, p. 17) explica este cambio mediante una regla de formación de prefijos que toma los morfemas libres como input y los convierte en prefijos. 
Señala Buenafuentes de la Mata (2001-02) que las formaciones con mal- deben considerarse formas prefijadas porque han desarrollado significados distintos de los que presenta el adverbio mal en la estructura sintáctica, contenidos que son propios de los prefijos: la negación ( malograr = 'no lograr') o la intensidad ( malherido $=$ 'herido gravemente', malcomer $=$ 'comer poco'). Del mismo modo, medio funciona como adjetivo (media docena) para designar la mitad de entidades representadas en el nombre al que modifica. A partir de este significado, ha desarrollado significados gradativos próximos a semi-, entre- o casiunido a nombres (medio novio, medio profesora), adjetivos (medio bobo, medio dormido) y verbos (medio enamorarse, medio llorar). En este uso gradativo, medio es una palabra átona y se convierte en un proclítico (Moliner 1968) que puede intercalarse entre el verbo auxiliar y la forma no personal del verbo en las formas compuestas y en las perífrasis ("Se ha medio enamorado", "Está medio llorando") (RAE y ASALE 2009). Por ello, no es extraño que medio haya sido considerado por varios autores como prefijo (Varela y Martín García 1999; RAE y ASALE 2009; Fábregas 2014; Felíu y Pato 2015). También el adverbio aspectual recién ("Recién llegó", "una cocina recién limpia”) puede presentar usos prefijales en formaciones como recienparida, recién nacido, (pan) recién hecho (Moliner 1968; Martín García y Varela 2007).

En el caso de la preposición, ya desde Alemany Bolufer (1920) se ha considerado que los prefijos eran fundamentalmente preposiciones y, por tanto, las formaciones resultantes debían ser consideradas como compuestos. Esta idea se ha mantenido con explicaciones distintas en los estudios que defienden una concepción sintáctica de la morfología (v.gr., Kornfeld y Saab 2003). Si bien estas explicaciones son válidas para las formaciones que conservan el significado locativo de la preposición, plantean problemas a la hora de estudiar las formaciones en las que el elemento inicial ha desarrollado valores adverbiales intensivos como prefijo (sobrevolar/ sobrecargar), lo que le permite funcionar como un operador (contradecir = 'decir algo con el fin de anular lo que se ha dicho antes'), denotar escalas de gradación ( sobrecargar = 'cargar mucho', entreabrir = 'abrir a medias') y seleccionar bases adjetivales (sobreagudo, entrefino). En consecuencia, las preposiciones también experimentan un proceso de gramaticalización que las convierte en prefijos.

El proceso de gramaticalización de una unidad puede entenderse asimismo como una pérdida de rasgos léxicos que tiene 
como consecuencia convertir un elemento dependiente léxico en un elemento dependiente más gramatical. Este proceso se da con mucha frecuencia en los prefijos del español. Según hemos señalado, los prefijos adjetivales calificativos pueden funcionar también como adverbios (macrovender, macroestético). Este cambio supone una pérdida de contenido léxico, puesto que el prefijo se convierte en simple operador de intensificación con distinto alcance: por ejemplo, macrovender es 'vender muchas cosas', no 'vender cosas grandes'. Este tipo de prefijos está ya muy gramaticalizado, razón por la cual hay cierta unanimidad en las gramáticas y en los estudios de morfología a la hora de considerarlos como prefijos.

El mismo proceso de gramaticalización está operando en las formas provenientes de adjetivos relacionales acortados (turismo ecológico > ecoturismo). Como los prefijos adjetivales calificativos, los acortamientos de adjetivos relacionales pueden adjuntarse a bases adjetivales (ecoagradable) y verbales (ecoexistir) con un valor adverbial que se aleja en muchos casos del adverbio procedente del adjetivo relacional. Así, si una formación como ecoturismo tiene un significado próximo a la construcción sintáctica turismo ecológico, los neologismos ecoagradable y ecoexistir no tienen el mismo significado de los sintagmas agradable ecológicamente o existir ecológicamente. Véase que estos acortamientos empiezan a tener un significado menos preciso, lo que les permite ser más recurrentes y productivos. Tales características justifican que las formas acortadas de los adjetivos relacionales se consideren prefijos. No obstante, no todos los prefijos adjetivales relacionales presentan el mismo grado de gramaticalización. Así, frente a eco-, ciber- o tecno-, euro- sólo es productivo unido a bases nominales (eurodiputado) ${ }^{7}$. En otros casos, la productividad y la recurrencia del prefijo es limitada. Por ejemplo, foto- como acortamiento de fotoeléctrico (célula fotoeléctrica $>$ fotocélula) aparece hasta el momento en pocas formaciones, frente a la productividad de foto- como elemento compositivo (fotoprotector) o como acortamiento de fotografía (fotomontaje). A diferencia de los prefijos adjetivales calificativos, los prefijos adjetivales

${ }^{7}$ Sólo hemos encontrado dos ejemplos de formaciones verbales recogidas en el artículo de García Palacios (2000-01): "Blair se euroenreda"; "El escritor alemán, que afirmó que hay que euronormalizar la literatura, recordó sus viajes a España”. 
relacionales conservan parte de su significado, lo que les impide funcionar como operadores o establecer escalas semánticas.

El proceso de gramaticalización que estamos describiendo es muy activo con adjetivos, adverbios y preposiciones y con los elementos dependientes que funcionan como estas categorías. Tal proceso no se da con los elementos compositivos, pues no existen elementos dependientes nominales ni acortamientos nominales que comiencen a funcionar como adjetivos y luego como adverbios. Por tanto, es el carácter nominal de un elemento el que se constituye como el límite entre los elementos compositivos y los prefijos, según defendemos en este trabajo.

Ahora bien, los prefijos no presentan un comportamiento homogéneo debido al distinto grado de gramaticalización. Como categoría, los prefijos son elementos modificadores con un contenido léxico poco especificado, lo que les permite funcionar como operadores y constituir escalas semánticas. En el proceso de gramaticalización, la pérdida de rasgos léxicos conlleva la de las restricciones de subcategorización, por lo cual una misma unidad puede modificar bases de distinta categoría. Esta pérdida de rasgos léxicos es lo que mejor define el comportamiento prefijal de un elemento y lo que explica las propiedades más prototípicas de un prefijo. Como señala Montermini (2008, p. 144), la productividad de un esquema derivativo aumenta con el grado de gramaticalización de los elementos que lo integran, idea que justifica que los prefijos sean más productivos que los elementos compositivos.

Otra propiedad que se deriva de esta pérdida de rasgos léxicos es el significado general que presentan los prefijos, lo que ha llevado a hablar en muchos casos de su polisemia ${ }^{8}$. Este significado general puede entenderse en muchos prefijos como un significado funcional: la negación, la gradación, el aspecto, la cuantificación, etc. Sin embargo, que un prefijo tenga un significado funcional no supone que las formaciones presenten opacidad semántica, sino que, por el contrario, es precisamente esta falta de un significado más léxico lo que confiere a las palabras prefijadas un significado más transparente.

Este camino de los prefijos hacia la expresión de contenidos más gramaticales está muy bien trazado en los prefijos intensi-

8 Algunos autores como KAGAN (2013) hablan de la polisemia de los prefijos a partir de un único significado funcional. Según esta autora, es la modificación de grado la característica fundamental de los prefijos. 
vos o gradativos, grupo reciente en el que se incluyen nuevos elementos (medio-, casi-) o elementos de otras clases en los que ha operado un cambio de significado: desde la locación (ultra-, super-, sobre-, infra-...), la iteración (re-), la dimensión (macro-, mega-, mini-...), la cuantificación (semi-, multi-) o la simple cualidad (seudo-). De este modo, prefijos que tenían un significado más especificado empiezan a confundirse con prefijos que sólo actúan como operadores de intensidad. Por ejemplo, en una formación como megamillonario, el prefijo mega- no denota el tamaño (su significado más específico), sino que actúa como otros prefijos intensivos: multimillonario o supermillonario. Del mismo modo, el prefijo seudo- no indica cualidad en una formación como seudorreligioso, sino más bien un grado inferior, significado próximo al que presentan otros prefijos intensivos (semirreligioso, casirreligioso).

\section{Conclusión}

Comenzamos este trabajo analizando las relaciones de modificación que se producen en el margen izquierdo de la palabra. Los elementos dependientes iniciales pueden modificar como sustantivos (dermoprotector), adjetivos (seudoproblema), adverbios (inmaduro), preposiciones (entretela) o cuantificadores (multicolor). Según hemos mostrado en este trabajo, es posible distinguir entre elementos compositivos y prefijos tomando como criterio la modificación de la base. Los elementos compositivos sólo pueden modificar como sustantivos frente a los prefijos, que pueden ser modificadores adjetivales, adverbiales, preposicionales y cuantificadores. Esta diferencia entre las dos categorías se ve corroborada por once criterios que ilustran el funcionamiento de los elementos compositivos y de los prefijos. Como hemos señalado, los elementos compositivos pueden ser argumentos del núcleo y se combinan con sufijos; ambas propiedades quedan descartadas de los prefijos. Asimismo, como las raíces, los elementos compositivos presentan un significado léxico específico, por lo cual no son polisémicos, tienen una selección categorial más limitada y muestran una menor productividad. Ante ellos, los prefijos tienen un significado más general, lo que facilita la selección de bases de distintas categorías, el desarrollo de diversos matices significativos según la base y, por tanto, el aumento de su productividad. Además, los 
prefijos pueden funcionar como operadores con distinto alcance y establecer escalas semánticas.

Si se relacionan los criterios que hemos utilizado para caracterizar los elementos compositivos y los prefijos con el modo de modificar de estas unidades, es posible establecer una escala funcional que va desde los elementos más léxicos (los que modifican como sustantivos) hasta los elementos más gramaticales (los que modifican como cuantificadores). Dado que los prefijos modifican la base como adjetivos, adverbios, preposiciones y cuantificadores, puede hablarse de tipos de prefijos según el modo de modificación, pero no de unidades distintas, porque obligaría a considerar un mismo elemento como dos categorías. Según hemos tratado de mostrar, a diferencia de los elementos compositivos, los prefijos tienen un significado poco especificado, lo que los habilita para establecer distintos tipos de modificación de la base. Tal caracterización nos permite incluir en esta categoría morfológica elementos que en algunas clasificaciones se han considerado elementos compositivos: por ejemplo, los prefijos adjetivales calificativos (seudo-, macro-, etc.) y las formas recientes derivadas de adjetivos relacionales acortados (eco-, ciber-, etcétera).

Los prefijos, como unidad morfológica, quedan definidos por su contenido léxico poco especificado, lo cual facilita su funcionamiento como categoría funcional y justifica sus propiedades más destacadas. Puesto que la pérdida de rasgos léxicos es gradual, los prefijos presentan diferencias en su comportamiento. La prefijación así entendida es un proceso distinto de la composición.

\section{REFERENCIAS}

Alemany Bolufer, José 1920. Tratado de la formación de palabras en la lengua castellana. La derivación y la composición. Estudio de sufijos empleados en una y otra, Librería de Victoriano Suárez, Madrid.

Amiot, Dany et Walter de Mulder 2002. "De l'adverbe au préfixe en passant par la préposition: un phénomène de grammaticalisation?”, Linguisticae Investigationes, 25, 2, pp. 247-272.

Bok-Bennema, Reineke 1993. "Native prefixoids in Spanish”, Language and Cognition, 3, pp. 11-20.

Buenafuentes de la Mata, Cristina 2001-02. "Procesos de gramaticalización en el estudio de los compuestos del español: el caso del elemento mal/a(-)", Anuari de Filologia, 23/24, 11/12, pp. 17-29. 
Bustos Gisbert, Eugenio 1986. La composición nominal en español, Ediciones Universidad de Salamanca, Salamanca.

Casado Velarde, Manuel 2015. La innovación léxica en el español actual, Síntesis, Madrid.

DEA = Manuel Seco et al. 2011 [1999]. Diccionario del español actual, Aguilar, Madrid.

$D L E=$ RAE y ASALE 2014. Diccionario de la lengua española, 23ª edición, Espasa-Calpe, Madrid.

FÁbregas, Antonio 2014. "Problemas de linearización: prefijos de resultado en español”, Lingüistica y Literatura, 65, pp. 65-85.

Felíu, Elena y Enrique Pato 2015. "Medio adverbio, medio prefijo: la evolución de medio como modificador de verbos en español", Boletín de la Real Academia Española, 95, 311, pp. 61-83.

García Palacios, JoaQuín 2000-01. "La quimera del euro-. Acerca de algunos elementos compositivos en español”, Revista de Lexicografía, 7, pp. 33-62, doi: 10.17979/rlex.2001.7.0.5602.

Gonçalves, Carlos A. y Katia Emmerick 2012: "El status de los componentes morfológicos y el continuum composición-derivación en portugués", Lingüistica, 28, pp. 119-145.

Heyna, Franziska 2012. Étude morpho-syntaxique des parasynthétiques. Les dérivés en "dé-" et en "anti-", Éditions Duculot, Bruxelles.

IACOBINI, Claudio 1997. "Distinguishing derivational prefixes from initial combining forms", Mediterranean Morphology Meetings, 1, pp. 132-140, doi: $10.26220 / \mathrm{mmm} .2350$.

Iglesias Cancela, Yolanda 2017. "Sobre los temas integrantes de los compuestos neoclásicos. Una propuesta de clasificación”, Verba, 44, pp. 481505.

Kagan, Olga 2013. "Scalarity in the domain of verbal prefixes", Natural Language and Linguistic Theory, 31, pp. 483-516, doi: 10.1007/s11049013-9190-z.

KORNFELD, LAURA 2009. "Los prefijos: propiedades sintácticas y morfofonológicas”, en De la palabra al texto. Estudios lingüísticos del español. Ed. Guiomar E. Ciapuscio, Eudeba, Buenos Aires, pp. 165-189.

Kornfeld, LaURa \& AndrÉs L. SAab 2003. "Morphology and syntax: Prepositional prefixes in Spanish", en Topics in morphology. Selected papers from the Third Mediterranean Morphology Meeting. Eds. Geert Booij, Janet DeCesaris, Angela Ralli \& Sergio Scalise, IULA, Barcelona, pp. 227-240. [Versión preprint].

Martín García, Josefa 1998. "Los prefijos intensivos del español: caracterización morfo-semántica”, Estudios de Lingüistica, 12, pp. 103-116, hdl: 10045/6331; doi: 10.14198/ELUA1998.12.07.

Martín García, Josefa 2017. "Los límites de la prefijación”, en Procesos morfológicos. Zonas de interferencia. Ed. Jesús Pena, Verba (Anexo 76), pp. 77-104, doi: 10.15304/9788416954292.

Martín García, Josefa y Soledad Varela 2007. "Naturaleza gramatical y valor semántico-aspectual de recién”, en Actas del VI Congreso de Lingüística General. Arco/Libros, Madrid, pp. 1733-1742.

Moliner, María 1968. Diccionario de uso del español, Gredos, Madrid. 
Montermini, Fabio 2008. Il lato sinistro della morfologia: la prefissazione in italiano e nelle lingue del mondo, Franco Angeli, Milano.

Olsen, Susan 2014. "Delineating derivation and compounding", en The Oxford Handbook of derivational morphology. Eds. Rochelle Lieber \& Pavol Stekauer, Oxford University Press, Oxford, pp. 26-49, doi: 10.1093/ oxfordhb/9780199641642.013.0003.

Prćıć, Tvrtko 2005. "Prefixes vs initial combining forms in English: A lexicographic perspective”, International Journal of Lexicography, 18, 3, pp. 313-334, doi: 10.1093/ijl/eci026.

RAE y ASALE 2009. Nueva gramática de la lengua española, Espasa-Calpe, Madrid.

Rainer, Franz 1993. Spanishe Wortbildungslehre, Niemeyer, Tübingen.

Rio-Torto, Graça 2014. "Prefixação e composição: fronteiras de um contínuo", Verba, 41, pp. 103-121.

Rio-Torto, Graça (coord.) 2013. Gramática derivacional do portugués, Coimbra University Press, Coimbra, doi: 10.14195/978-989-26-0864-8.

Val Álvaro, José F. 1999. "La composición”, en Gramática descriptiva de la lengua española. Dirs. Ignacio Bosque y Violeta Demonte, Espasa Calpe, Madrid, t. 3, pp. 4757-4842.

Varela, Soledad y Josefa Martín García 1999. "La prefijación”, en Gramática descriptiva de la lengua española. Dirs. Ignacio Bosque y Violeta Demonte, Espasa Calpe, Madrid, t. 3, pp. 4993-5040. 
